\title{
CCND1 G870A polymorphism contributes to the risk of esophageal cancer: An updated systematic review and cumulative meta-analysis
}

\author{
LI WEN ${ }^{1}$, YUAN-YUAN HU ${ }^{2}$, GONG-LI YANG ${ }^{3}$ and DE-XI LIU ${ }^{1}$ \\ Departments of ${ }^{1}$ Dermatology, and ${ }^{2}$ Stomatology and Evidence-Based Medicine Center, Taihe Hospital, \\ Hubei University of Medicine, Shiyan 442000; ${ }^{3}$ Department of Gastroenterology, Nanfang Hospital, \\ Southern Medical University, Baiyun, Guangzhou 510515, P.R. China
}

Received March 7, 2014; Accepted May 14, 2014

DOI: 10.3892/br.2014.286

\begin{abstract}
The common functional cyclin D1 (CCND1) G870A polymorphism may influence the risk of esophageal cancer. However, the conclusions of previous studies have been inconsistent for the association between the CCND1 G870A polymorphism and esophageal cancer risk. A meta-analysis of 11 published case-control studies was performed, including 2,111 patients with esophageal cancer and 3,232 controls, to investigate the association between the CCND1 G870A polymorphism and esophageal cancer risk. The odds ratio (OR) with a 95\% confidence interval (CI) was applied to assess the association between the CCND1 G870A polymorphism and esophageal cancer risk. A significant association between the CCND1 G870A polymorphism and esophageal cancer risk was observed for the allele contrast (A vs. G: OR, 1.23; 95\% CI, 1.02-1.48; $\mathrm{P}=0.029$ ), codominant (AA vs. GG: OR, $1.58 ; 95 \% \mathrm{CI} ; 1.06-2.35 ; \mathrm{P}=0.024)$ and recessive models (AA vs. $\mathrm{GG}+\mathrm{GA}$ : OR, 1.33, 95\% CI, 1.03-1.73; $\mathrm{P}=0.030$ ). However, in the stratified analysis by ethnicity, study design and pathology, there was no significant association detected in these genetic models. In conclusion, results of the meta-analysis suggested that the CCND1 G870A polymorphism is a potential risk factor in the development of esophageal cancer.
\end{abstract}

\section{Introduction}

Esophageal cancer is one of the most common types of malignant disease, with $\sim 16,980$ new cases and 14,710 mortalities in the United States in 2011 (1). Esophageal

Correspondence to: Dr Yuan-Yuan $\mathrm{Hu}$, Department of Stomatology and Evidence-Based Medicine Center, Taihe Hospital, Hubei University of Medicine, 32 South Renmin Road, Shiyan 442000, P.R. China

E-mail: hyy19820922@yeah.net

Key words: CCND1, polymorphism, esophageal cancer, meta-analysis cancer is a multifactorial disease, which is considered to be a result of complex interactions between environmental and genetic factors. Diet has been hypothesized to play a role in the etiology of esophageal cancer. A number of studies have found that consuming large quantities of red or processed meat is associated with an increase in the risk of esophageal cancer (2-4).

Aberrant cell proliferation is an important factor for the development of numerous types of common cancer. Cyclin families are involved in cell-cycle progression, and in particular, cyclin D1 (CCND1) is the major regulatory protein that plays a key role in the transition from $\mathrm{G} 1$ to $\mathrm{S}$ phase by binding to cyclin-dependent kinases 4 and 6 to promote the progression of the cell cycle during cell division $(5,6)$. The overexpression of CCND1 has always been observed in numerous types of malignant cancer and indicates a poor clinical outcome (7-9).

Single-nucleotide polymorphisms (SNPs) may change the functions of the gene and alter the protein expression, potentially affecting cell proliferation and increase the susceptibility of developing cancer. The synonymous SNP (rs603965) of a $\mathrm{G}$ to A polymorphism at codon 242 (G870A) in exon 4, is the most important mutation of the CCND1 gene. The A allele creates a greater frequency of alternate splicing during transcription, which was postulated to have a longer half life than the $\mathrm{G}$ allele to bypass the G1/S checkpoint and resulted in an increased CCND1 level, leading to abnormal cell proliferation and circumvention of apoptosis $(10,11)$.

Although a number of epidemiological studies have been conducted to assess the association between the CCND1 G870A polymorphism and esophageal cancer susceptibility, the conclusions have been inconsistent. Thus far, two related meta-analyses were conducted by Cai et al (12) and Zhou et al (13), which demonstrated various associations between the CCND1 G870A polymorphism and esophageal cancer risk. Notably, the meta-analyses by Cai et al (12) only included eight published studies, and the study by Zhou et al (13) was conducted with a focus on Asian populations only. In the present study, 11 case-control studies on the CCND1 G870A polymorphism and esophageal cancer risk that were previously published were analyzed by performing a meta-analysis to examine a more specific association between 
the CCND1 G870A polymorphism and esophageal cancer risk and various published observational studies.

\section{Materials and methods}

Search strategy. The Pubmed database was searched using the terms 'CCND1', 'cyclin D1', 'esophageal cancer', 'polymorphism', and the combined phrases for all genetic studies on the association between the CCND1 G870A polymorphism and esophageal cancer risk between 2003 and March 7, 2014. Furthermore, the search was complemented with a examination of the references of the retrieved studies and reviews. The following criteria were used to select the studies for the meta-analysis: i) Observational (case-control or prospective) studies of the CCND1 G870A polymorphism and esophageal cancer risk; ii) sufficient published data for estimating an odds ratio (OR) with a 95\% confidence interval (CI); and iii) if studies had partly or overlapping data, only the largest or most recent sample was selected, according to Little et al (14). A total of 11 case-control studies, including 2,111 patients with esophageal cancer and 3,232 controls, were included in this meta-analysis.

Data extraction. Data were extracted independently by two investigators (Wen and $\mathrm{Hu}$ ) from all the selected studies. The data included the first author's name, publication data, country of origin, sources of controls, ethnicity of the study population (categorized as Asian, Caucasian and Mixed) and number of different genotype, tumor pathology and Hardy-Weinberg equilibrium (HWE) in controls.

Statistical analysis. The allele contrast (A vs. G) and codominant (AA vs. GG, GA vs. GG), dominant [(AA+GA) vs. GG) and recessive models (AA vs. (GG+GA)] were evaluated using ORs with $95 \% \mathrm{CI}$ to assess the strength of the association between the CCND1 G870A polymorphisms and esophageal cancer risk. Subgroup statistical analyses were conducted for ethnicity, study design and pathology. Otherwise, heterogeneity and cumulative analysis were assessed by $\chi^{2}$-based Q-test (15). OR estimation was calculated with the fixed-effect model (Mantel-Haenszel method) when statistical heterogeneity did not exist $(\mathrm{P}>0.10)(16)$. Otherwise, the random-effects model (DerSimonian and Laird method) was selected (17). Publication bias was evaluated by the Begg's funnel plot and linear regression asymmetry test by Egger et al $(18,19)$. Statistical analysis was performed using STATA versions 10.0 and 11.0 (StataCorp, College Station, TX, USA), and two-sided $\mathrm{P}$-values $(\mathrm{P}<0.05)$ were considered to indicate a statistically significant difference.

\section{Results}

Study characteristics. A total of 73 relevant studies were identified (Fig. 1). Following a careful review, 10 published studies with 11 case-control studies were identified, with 2,111 patients esophageal cancer patients and 3,232 controls (20-29). The distribution of the various genotypes of each study in different populations is shown in Table I. The diverse genotyping methods were polymerase chain reaction-restriction fragment length polymorphism, highly parallel SNP genotyping

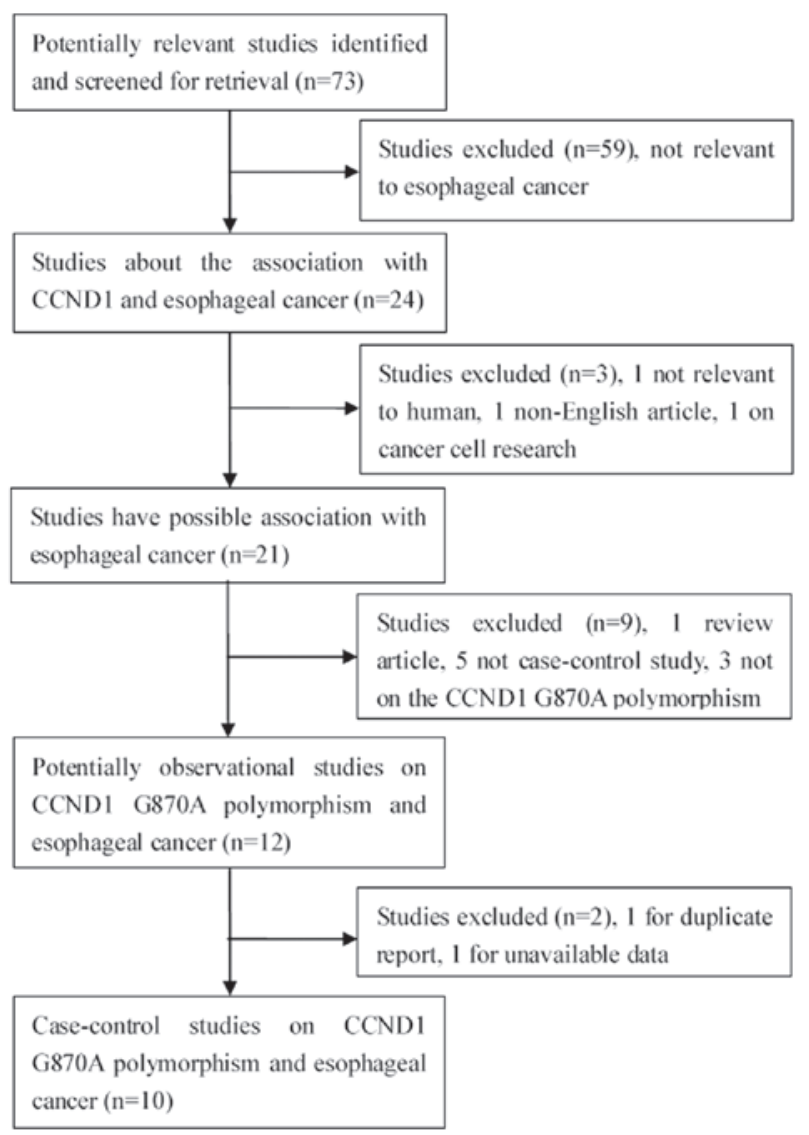

Figure 1. Flow diagram of the study selection process.

assay and Taqman techniques. No study deviated from Hardy-Weinberg equilibrium (HWE) in control populations.

Meta-analysis. The main results of the meta-analysis and the heterogeneity test are shown in Table II. Overall, there was a significant association between the CCND1 G870A polymorphism and esophageal cancer risk was observed for the allele contrast (A vs. G: OR, 1.23; 95\% CI, 1.02-1.48; $\mathrm{P}=0.029 \mathrm{P}_{\text {heterogeneity }}<0.01$ ), codominant (AA vs. GG: OR, 1.58; 95\% CI, 1.06-2.35; $\left.\mathrm{P}=0.024, \mathrm{P}_{\text {heterogeneity }}<0.01\right)$ and recessive models [AA vs. $(\mathrm{GG}+\mathrm{GA})$ : OR, $1.33,95 \% \mathrm{CI}, 1.03-1.73$; $\mathrm{P}=0.030, \mathrm{P}_{\text {heterogeneity }}<0.01 ;$ Fig. 2]. Simultaneously, a borderline significant increased risk was found in the dominant model [(AA + GA) vs. GG: OR, 1.30, 95\% CI, 0.96-1.76; $\mathrm{P}=0.092$, $\left.\mathrm{P}_{\text {heterogeneity }}<0.01\right]$. No significant risk effect was found in the subgroup analysis by ethnicity, study design and pathology.

Publication bias. The Begg's funnel plot and Egger's test were used to estimate the publication bias in the five models. The shape of the funnel plots appeared to be symmetrical in the GA vs. GG and (AA + GA) vs. GG models, but not in the A vs. G, AA vs. GG and AA vs. (GG + GA) models (Fig. 3), indicating that there was a certain amount of publication bias. Egger's test was applied to provide further statistical evidence $[(\mathrm{P}=0.001$ for $\mathrm{A}$ vs. $\mathrm{G} ; \mathrm{P}<0.001$ for $\mathrm{AA}$ vs. $\mathrm{GG}$; and $\mathrm{P}<0.001$ for $A A$ vs. $(G G+G A)]$.

Cumulative and sensitivity analysis. Each study that was involved in the meta-analysis was deleted separately to assess 
Table I. Characteristics of the case-control studies included in the meta-analysis.

\begin{tabular}{|c|c|c|c|c|c|c|c|c|c|c|c|c|c|c|}
\hline \multirow[b]{3}{*}{ First author } & \multirow[b]{3}{*}{ Year } & \multirow{3}{*}{$\begin{array}{l}\text { Country } \\
\text { /region }\end{array}$} & \multirow{3}{*}{$\begin{array}{c}\text { Racial } \\
\text { descent }\end{array}$} & \multirow{3}{*}{$\begin{array}{l}\text { Source of } \\
\text { controls }\end{array}$} & \multirow[b]{3}{*}{ Case, $\mathrm{n}$} & \multirow[b]{3}{*}{ Control, $\mathrm{n}$} & \multicolumn{6}{|c|}{ Genotype distribution } & \multirow{3}{*}{$\begin{array}{c}\text { P-value } \\
\text { HWE }^{\mathrm{a}}\end{array}$} & \multirow[b]{3}{*}{ Pathology } \\
\hline & & & & & & & \multicolumn{3}{|c|}{ Case, $\mathrm{n}$} & \multicolumn{3}{|c|}{ Control, $\mathrm{n}$} & & \\
\hline & & & & & & & GG & GA & $\mathrm{AA}$ & GG & GA & AA & & \\
\hline Yu & 2003 & China & Asian & Population-based & 321 & 345 & 68 & 157 & 96 & 58 & 177 & 110 & 0.354 & ESCC \\
\hline Zhang & 2003 & China & Asian & Population-based & 120 & 183 & 11 & 74 & 35 & 38 & 102 & 43 & 0.118 & ESCC \\
\hline Casson & 2005 & Canada & Caucasian & Hospital-based & 56 & 95 & 12 & 27 & 17 & 35 & 52 & 8 & 0.063 & EA \\
\hline Geddert & 2005 & Germany & Caucasian & Hospital-based & 56 & 253 & 16 & 26 & 14 & 63 & 136 & 54 & 0.224 & EA \\
\hline Jain & 2007 & India & Asian & Hospital-based & 151 & 201 & 22 & 76 & 53 & 37 & 111 & 53 & 0.114 & Mixed \\
\hline Akbari $^{\mathrm{b}}$ & 2009 & Iran & Caucasian & NA & 279 & 807 & 72 & 126 & 81 & 161 & 376 & 270 & 0.149 & ESCC \\
\hline Akbari $^{\mathrm{c}}$ & 2009 & Iran & Caucasian & NA & 465 & 561 & 97 & 238 & 130 & 107 & 290 & 164 & 0.290 & ESCC \\
\hline Liu & 2010 & USA & Caucasian & Hospital-based & 299 & 450 & 79 & 154 & 66 & 128 & 215 & 107 & 0.369 & EA \\
\hline Kurmanov & 2010 & Kazakhstan & Caucasian & Population-based & 98 & 86 & 19 & 42 & 37 & 24 & 46 & 16 & 0.463 & ESCC \\
\hline Hussain & 2011 & India & Asian & Population-based & 151 & 151 & 20 & 99 & 32 & 56 & 72 & 23 & 0.986 & ESCC \\
\hline Djansugurova & 2013 & Kazakhstan & Caucasian & Healthy-based & 115 & 100 & 22 & 49 & 44 & 28 & 54 & 18 & 0.363 & ESCC \\
\hline
\end{tabular}

${ }^{a} \mathrm{HWE}$ in controls; ${ }^{\mathrm{b}}$ study 1; ' study 2. HWE, Hardy-Weinberg equilibrium; ESCC, esophageal squamous cell carcinoma; EA, esophageal adenocarcinoma; Mixed, ESCC and EA; NA, not available.

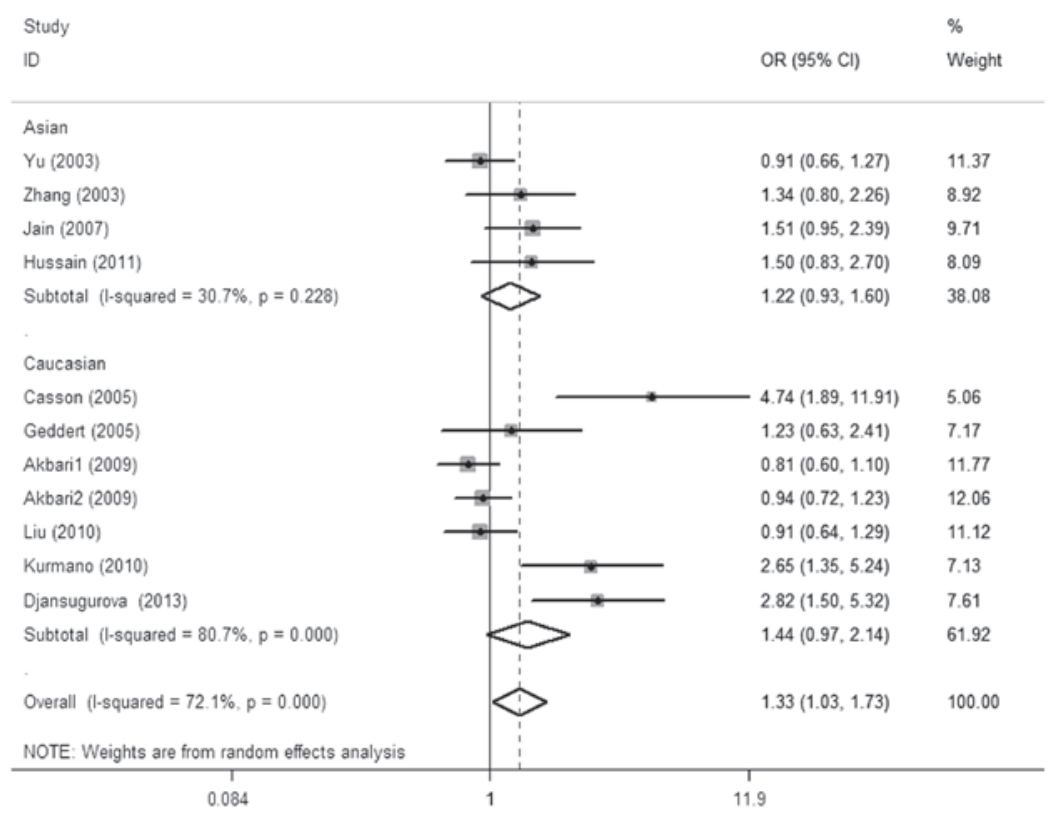

Figure 2. OR of esophageal cancer associated with CCND1 G870A polymorphism for the AA vs. GG + GA model in total. OR, odds ratio; CI, confidence interval; CCND1, cyclin D1.

the influence of the individual dataset to the pooled ORs. The analysis results demonstrated a slightly decreased effect each time.In the cumulative meta-analysis, the results did not become significant until the last study by Djansugurova et al (29) was accumulated (Fig. 4). Furthermore, the analysis results demonstrated that there was no significant association when the study of Djansugurova et al (29) was removed.

\section{Discussion}

CCND1 has been mapped to chromosome 11q13, encoding a key cell cycle regulatory protein with 295 amino acids. CCND1 regulates the transition from the $\mathrm{G} 1$ to $\mathrm{S}$ phase

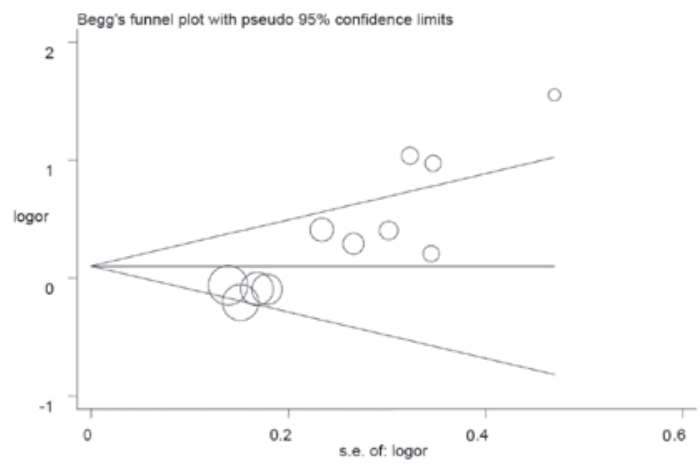

Figure 3. Begg's funnel plot analysis was used to detect publication bias for the AA vs. GG + GA model. Each point represents a separate study. 


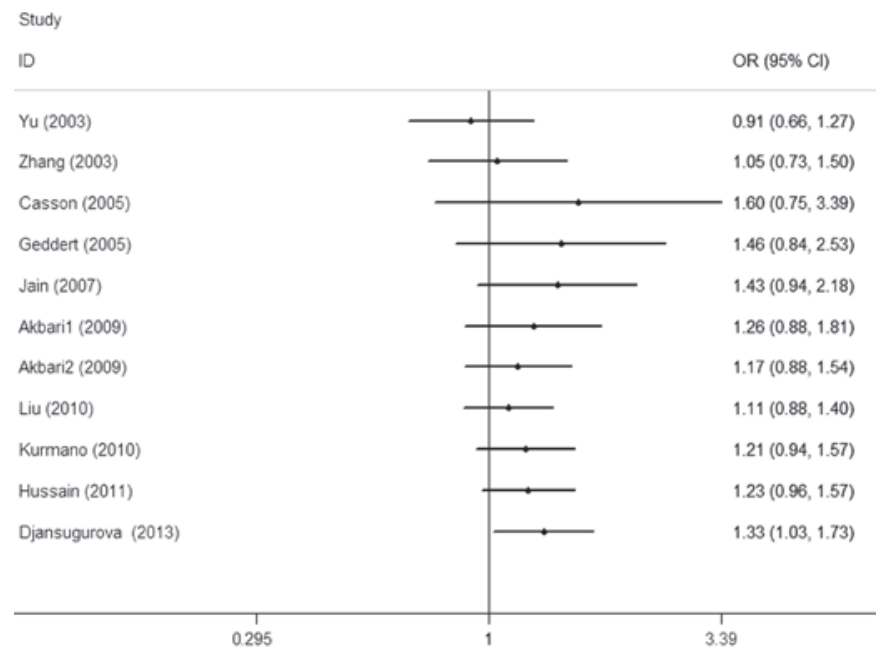

Figure 4. Cumulative meta-analyses according to the publication year in AA vs. GG + GA model.

during cell division. A high activity of CCND1 results in the premature cell passage through the G1-S transition, which leads to the generation of unrepaired DNA damage and the accumulation of genetic errors (30). The protein overexpression of CCND1 has been found in numerous types of cancer, and has also been regarded as the malignant characterization of cancer. There are various polymorphisms in CCND1, but the $\mathrm{G}$ to A mutation is well known and does not result in any amino acid alteration within the protein sequence. However, the CCND1 A allele results in an alternatively spliced transcript of CCND1 with a longer half life than the CCND1 $\mathrm{G}$ allele. This mutation helps the variant cell pass through the G1/S checkpoint easily and results in abnormal perforation, leading to cancer development (31). Findings of previous studies have shown that the CCND1 A allele may increase the risk of breast, prostate, colorectal and other types of cancer in different ethnicities (32-36).

In 2003, Yu et al (20) conducted the first study between the CCND1 G870A polymorphism and esophageal cancer, however, no significant association was found in a Chinese population. Thus far, conflicting conclusions on the association of the CCND1 G870A polymorphism and esophageal cancer susceptibility exist. The study by Zhang et al (21) found an increased risk for developing esophageal cancer with the CCND1 870A allele, and 2.0 -fold increased risk was found among the AA genotype compared to the GG and GA genotypes in a Northern Chinese population. The study by Casson et al (22) found that the apparently elevated risk of esophageal cancer was associated with the AA genotype compared to the GG genotype (OR, 5.99; 95\% CI, 1.89-18.96) in a Canadian population. In the study by Jain et al (24), the AAgenotype was marginally associated with esophageal cancer (OR, 1.5; 95\% CI, 0.98-2.4), and there was a higher risk in the upper location (OR, 3.8; 95\% CI, 1.6-9.3) in an Indian population. Kurmano et al (26) reported a significantly increased association in Kazakhstan with the variant homozygous AA genotype (OR, 2.66; 95\% CI, 1.35-5.24). Hussain et al (27) also indicated that the Indian individuals carrying the GA and AA genotype had a 2.8-fold increased risk for the development of esophageal cancer, and the higher risk was observed in 
individuals with smoking and drinking habits. Conversely, the study by Akbari et al (28) found that the G allele was associated with a 1.5-fold increased risk of esophageal cancer under the recessive model (OR, 1.50; 95\% CI, 1.14-2.16; $\mathrm{P}=0.02$ ). Furthermore, no significant association between the CCND1 G870A polymorphism and esophageal cancer in a German population was found in the study by Geddert et al (23). A similar conclusion was also found in the study by Liu et al in an American population (25), and Djansugurova et al (29) in Kazakhstan. Recently, two meta-analyses were conducted by Cai et al (37) and Zhou et al (38), which demonstrated different conclusions in regards to the CCND1 G870A polymorphism with limited published data. Therefore, it is assumed that the reason for the contrary results may be the sample sizes. Thus far, a number of other studies $(28,38)$ regarding this focus have been published. Therefore, the meta-analysis was performed to clarify the results on the association. In the meta-analysis, including 11 case-control studies with 2,111 esophageal cancer patients and 3,232 controls, certain possible risks were explored between the potential function of the CCND1 G870A polymorphism and esophageal cancer.

There were several limitations for the analysis in the present study. Firstly, the results were based on the unadjusted estimates with unavailable original data of these collected studies, which limited the evaluation with certain covariates, including age, smoking, drinking and other environmental factors. Secondly, the sample size was relatively small in the analysis, which may have induced the bias of the results and the disability of drawing more detailed conclusions. Thirdly, the controls of several studies were hospital-based individuals with other diseases, which may result in specific selection biases. Fourthly, a certain amount of publication bias was always present until the subgroup analyses were conducted, similar to the sensitivity analysis. These deviations influenced the preciseness and reliability of the results. Additionally, the majority of the included studies were conducted on Caucasians and Asians, but there were no studies of African populations. Therefore the variation in ethnicity may have generated biases.

Taken together, despite these limitations, the meta-analysis suggests that the CCND1 G870A polymorphism is a potential elevated risk in the development of esophageal cancer. These findings may be helpful in increasing the understanding of the CCND1 G870A polymorphism in the etiology of esophageal cancer. In the future, large and well-designed case-control studies are required to verify these findings.

\section{Acknowledgements}

The authors gratefully acknowledge the support of the subjects who participated in the present study. The study was partly supported by the Foundation of Ministry of Education of Hubei Province (grant no. D20142407), Foundation of Hubei University of Medicine (grant no. 2013GPY07) and Taihe Hospital (grant nos. EBM2013006 and EBM2013031).

\section{References}

1. Siegel R, Ward E, Brawley O and Jemal A: Cancer statistics, 2011: the impact of eliminating socioeconomic and racial disparities on premature cancer deaths. CA Cancer J Clin 61: 212-236, 2011
2. Keszei AP, Schouten LJ, Goldbohm RA and van den Brandt PA: Red and processed meat consumption and the risk of esophageal and gastric cancer subtypes in The Netherlands Cohort Study. Ann Oncol 23: 2319-2326, 2012.

3. Steevens J, Schouten LJ, Goldbohm RA and van den Brandt PA: Vegetables and fruits consumption and risk of esophageal and gastric cancer subtypes in the Netherlands cohort study. Int J Cancer 129: 2681-2693, 2011.

4. Bravi F, Edefonti V, Randi G, et al: Dietary patterns and the risk of esophageal cancer. Ann Oncol 23: 765-770, 2012.

5. Sherr CJ: Cancer cell cycles. Science 274: 1672-1677, 1996.

6. Zhao J, Li L, Wei S, et al: Clinicopathological and prognostic role of cyclin D1 in esophageal squamous cell carcinoma: a meta-analysis. Dis Esophagus 25: 520-526, 2011.

7. Kaminagakura E, Werneck da Cunha I, Soares FA, Nishimoto IN and Kowalski LP: CCND1 amplification and protein overexpression in oral squamous cell carcinoma of young patients. Head Neck 33: 1413-1419, 2011.

8. Troncone G, Volante M, Iaccarino A, et al: Cyclin D1 and D3 overexpression predicts malignant behavior in thyroid fine-needle aspirates suspicious for Hurthle cell neoplasms. Cancer 117: 522-529, 2009.

9. Abramson VG, Troxel AB, Feldman M, et al: Cyclin D1b in human breast carcinoma and coexpression with cyclin D1a is associated with poor outcome. Anticancer Res 30: 1279-1285, 2010.

10. Betticher DC, Thatcher N, Altermatt HJ, Hoban P, Ryder WD and Heighway J: Alternate splicing produces a novel cyclin D1 transcript. Oncogene 11: 1005-1011, 1995.

11. Li Z, Jiao X, Wang C, et al: Alternative cyclin D1 splice forms differentially regulate the DNA damage response. Cancer Res 70: 8802-8811, 2010.

12. Cai W, Wang ZT, Zhong J and Zhang Y: Lack of association between Cyclin D1 gene G870A polymorphism and esophageal cancer: evidence from a meta-analysis. Genet Mol Res 12: 6636-6645, 2013.

13. Zhou X, Gu Y and Zhang SL: Association between p53 codon 72 polymorphism and cervical cancer risk among Asians: a HuGE review and meta-analysis. Asian Pac J Cancer Prev 13: 4909-4914, 2012.

14. Little J, Bradley L, Bray MS, et al: Reporting, appraising, and integrating data on genotype prevalence and gene-disease associations. Am J Epidemiol 156: 300-310, 2002.

15. Lau J, Ioannidis JP and Schmid CH: Quantitative synthesis in systematic reviews. Ann Intern Med 127: 820-826, 1997.

16. Mantel $\mathrm{N}$ and Haenszel W: Statistical aspects of the analysis of data from retrospective studies of disease. J Natl Cancer Inst 22: 719-748, 1959.

17. DerSimonian R and Laird N: Meta-analysis in clinical trials. Control Clin Trials 7: 177-188, 1986.

18. Harbord RM, Egger M and Sterne JA: A modified test for small-study effects in meta-analyses of controlled trials with binary endpoints. Stat Med 25: 3443-3457, 2006.

19. Egger M, Davey Smith G, Schneider M and Minder C: Bias in meta-analysis detected by a simple, graphical test. BMJ 315: 629-634, 1997.

20. Yu C, Lu W, Tan W, et al: Lack of association between CCND1 G870A polymorphism and risk of esophageal squamous cell carcinoma. Cancer Epidemiol Biomarkers Prev 12: 176, 2003.

21. Zhang J, Li Y, Wang R, et al: Association of cyclin D1 (G870A) polymorphism with susceptibility to esophageal and gastric cardiac carcinoma in a northern Chinese population. Int J Cancer 105: 281-284, 2003.

22. Casson AG, Zheng Z, Evans SC, et al: Cyclin D1 polymorphism (G870A) and risk for esophageal adenocarcinoma. Cancer 104: 730-739, 2005.

23. Geddert H, Kiel S, Zotz RB, et al: Polymorphism of p16 INK4A and cyclin D1 in adenocarcinomas of the upper gastrointestinal tract. J Cancer Res Clin Oncol 131: 803-808, 2005.

24. Jain M, Kumar S, Lal P, Tiwari A, Ghoshal UC and Mittal B: Role of BCL2 (ala43thr), CCND1 (G870A) and FAS (A-670G) polymorphisms in modulating the risk of developing esophageal cancer. Cancer Detect Prev 31: 225-232, 2007.

25. Liu G, Cescon DW, Zhai R, et al: p53 Arg72Pro, MDM2 T309G and CCND1 G870A polymorphisms are not associated with susceptibility to esophageal adenocarcinoma. Dis Esophagus 23: 36-39, 2010.

26. Kurmano BK, Djansugurova L, Bersimba R, et al: TP53 Arg72Pro and CCND1 A870G polymorphisms and esophageal cancer risk. J Life Sci 4: 16-20, 2010. 
27. Hussain S, Yuvaraj M, Thakur N, et al: Association of cyclin D1 gene polymorphisms with risk of esophageal squamous cell carcinoma in Kashmir Valley: a high risk area. Mol Carcinog 50: 487-498, 2011.

28. Akbari MR, Malekzadeh R, Shakeri R, et al: Candidate gene association study of esophageal squamous cell carcinoma in a high-risk region in Iran. Cancer Res 69: 7994-8000, 2009.

29. Djansugurova LB, Perfilyeva AV, Zhunusova GS, Djantaeva KB Iksan OA and Khussainova EM: The determination of genetic markers of age-related cancer pathologies in populations from Kazakhstan. Front Genet 4: 70, 2013.

30. Hall $M$ and Peters G: Genetic alterations of cyclins, cyclin-dependent kinases, and Cdk inhibitors in human cancer. Adv Cancer Res 68: 67-108, 1996.

31. Solomon DA, Wang Y, Fox SR, et al: Cyclin D1 splice variants. Differential effects on localization, RB phosphorylation, and cellular transformation. J Biol Chem 278: 30339-30347, 2003.

32. Sergentanis TN and Economopoulos KP: Cyclin D1 G870A polymorphism and breast cancer risk: a meta-analysis comprising 9,911 cases and 11,171 controls. Mol Biol Rep 38: 4955-4963, 2011.
33. Comstock CE, Augello MA, Benito RP, et al: Cyclin D1 splice variants: polymorphism, risk, and isoform-specific regulation in prostate cancer. Clin Cancer Res 15: 5338-5349, 2009.

34. Yaylim-Eraltan I, Arikan S, Yildiz Y, et al: The influence of cyclin D1 A870G polymorphism on colorectal cancer risk and prognosis in a Turkish population. Anticancer Res 30: 2875-2880, 2010.

35. Tsai MH, Tsai CW, Tsou YA, Hua CH, Hsu CF and Bau DT: Significant association of cyclin D1 single nucleotide polymorphisms with oral cancer in taiwan. Anticancer Res 31: 227-231, 2011.

36. Akkiz H, Bayram S, Bekar A, Akgöllü E and Ozdil B: Cyclin D1 G870A polymorphism is associated with an increased risk of hepatocellular carcinoma in the Turkish population: case-control study. Cancer Epidemiol 34: 298-302, 2010.

37. Cai W, Wang ZT, Zhong J and Zhang Y: Lack of association between Cyclin D1 gene G870A polymorphism and esophageal cancer: evidence from a meta-analysis. Genet Mol Res 12: 6636-6645, 2013.

38. Zhuo W, Zhang L, Wang Y, Zhu B and Chen Z: Cyclin D1 G870A polymorphism is a risk factor for esophageal cancer among Asians. Cancer Invest 30: 630-636, 2012. 